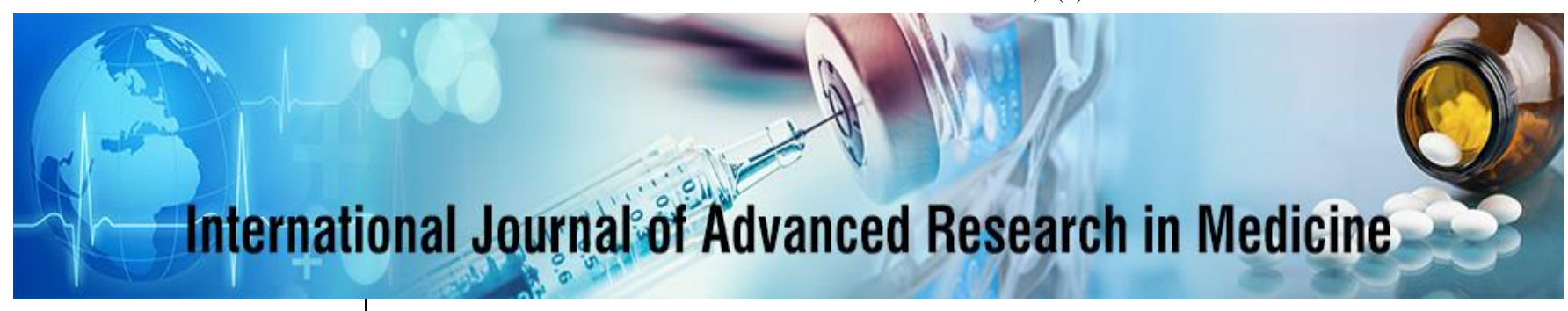

E-ISSN: 2706-9575

P-ISSN: 2706-9567

IJARM 2020; 2(1): 63-66

Received: 30-11-2020

Accepted: 31-12-2020

Dr. Arun Kumar Verma MD Medicine, Metro hospital and cancer research Center, Jabalpur, Madhya Pradesh, India

\section{To determine the correlation between HbA1c and AIP in patients diagnosed with type 2 diabetes mellitus}

\author{
Dr. Arun Kumar Verma
}

DOI: https://doi.org/10.22271/27069567.2020.v2.i1a.122

\begin{abstract}
Aim: to assess the lipid patterns and association of AIP with HbA1c in diabetes patients.

Methods: This study conducted in the Department of Medicine, Metro Hospital and Cancer Research Centre, Jabalpur, MP, India from October 2018 to January 2020. Total 160 diagnosed cases of DM type 2 in the age group of 20-70 years were included in this study. The patients were divided into 2 groups depending on the levels of glycated hemoglobin. Venous blood samples from all the subjects were collected after at least 6 hours fasting and analyzed for fasting plasma glucose (FPG), 2 hours post prandial glucose levels(2hPG), serum total cholesterol (TC), triglycerides (TG), HDL-C, Very low density lipoprotein cholesterol (VLDL-C) \& LDL-C analysis was done by standard methods. The atherogenic index of plasma (AIP) was calculated by the formula base 10 logarithm of the ratio of TG to HDL-C.

Results: Out of the 160 patients studied, there were 70 males and 90 females. The maximum number of patients was in the age group of 50-60 years. The levels of FBG, TC, TG,VLDL-C LDL-C, HbA1c, AIP, ratios of TC/HDL-C and LDL-C/HDL-C are increased, while the levels of HDL-C are decreased in patients with $\mathrm{HbAlc}>7 \%$ as compared to patients with $\mathrm{HbA} 1 \mathrm{c} \leq 7 \%$ and these values are highly significant. The patients with $\mathrm{HbA} 1 \mathrm{c}>7 \%$ had dyslipidemia as the most prominent feature in the lipid profile. Direct and significant correlation of $\mathrm{HbAlc}$ with FBG, 2Hpg was found.

Conclusion: It can be concluded from the present study that patients with poor glycaemic control have a atherogenic lipid profile and Glycated haemoglobin predicts dyslipidemia and atherogenicity.
\end{abstract}

Keywords: Lipid profile, type 2 diabetes mellitus, Glycosylated haemoglobin, glycaemic control, atherogenic index of plasma

\section{Introduction}

Diabetes is a global endemic with rapidly increasing prevalence in both developed and developing countries ${ }^{[1]}$. DM is a group of metabolic disease characterized by hyperglycemia resulting from defects in insulin secretion, insulin action or both. Uncontrolled diabetic patients are characterized by hyperglycemia, hyperinsulinemia, protein glycation and oxidative stress which cause early appearance of diabetic complications. The chronic hyperglycemia is associated with long-term damage dysfunction, and failure of various organs, especially the eyes, kidneys, nerves, heart and blood vessels ${ }^{[2]}$. There is a high risk of cardiovascular disease (CVD) in People with type 2 diabetes; Diabetic patients with accompanied dyslipidemia are silent targets for cardiovascular deaths which represent the top killer in this population ${ }^{[3]}$. Diabetes mellitus is a common secondary cause of hyperlipidaemia, particularly, if glycaemic control is poor, which in-turn is an important risk factor for atherosclerosis and coronary heart disease. Diabetes mellitus increases the risk of atherosclerotic vascular disease because of associated dyslipidemia, exhibit an atherogenic lipid profile, which greatly increases their CVD risk compared with people without diabetes. Glycosylated haemoglobin $(\mathrm{HbAlc})$ is commonly used as a marker of glycaemic status. Glycated haemoglobin $(\mathrm{HbA} 1 \mathrm{c})$ was called as unusual haemoglobin in patients with diabetes when it was first discovered. After that discovery, it was established that HbA1c could be used as an objective measure of glycaemic control and a validated relationship between A1C and average glucose across a range of diabetes types and patient populations by an International Expert Committee recommendation which was later adopted by WHO (International Expert Committee, 2009; World Health Organisation, 2011). HbA1c has been proposed as a dual marker for glycaemic control and coronary artery disease (CAD) risk factor ${ }^{[4]}$. The American diabetes association (ADA) estimates that the risk of diabetes-
Dr. Arun Kumar Verma MD Medicine, Metro hospital and cancer research Center, Jabalpur, Madhya Pradesh, India 
related mortality increases $25 \%$ for each $1 \%$ increase in $\mathrm{HbA} 1 \mathrm{c}$. It has also been estimated that each percentage point increase in $\mathrm{HbA} 1 \mathrm{c}$ correspond to a $35 \%$ increase in the risk of micro vascular complications and an $18 \%$ increase in the risk of myocardial infarction (fatal and non-fatal). The reduction or control of blood glucose level may lower the lipid risk factor for cardiovascular diseases [5]. There is evidence of close relationship between poor glycemic control and progression of dyslipidemia ${ }^{[6]}$. Most frequent complication of DM is atherosclerosis; it affects major vascular beds leading to various metabolic abnormalities. Intensive glycemic control means the glycohaemoglobin (HbA1c) or blood glucose values are normal or near normal range, no matter how simple or complex the treatment regimen. A strong correlation has been shown between lipid profile and $\mathrm{CAD}$. The Framingham study has demonstrated a linear increase in CAD risk with increment of TC level from $180 \mathrm{mg}$ upward. The study established that individuals with $\mathrm{HDL}$ cholesterol less than $35 \mathrm{mg} / \mathrm{dl}$ have 8 times increase in CAD incidence than those with HDL cholesterol more than $65 \mathrm{mg} / \mathrm{dl}{ }^{[7]}$. The Lipid Research clinics Coronary Primary Prevention Trial concluded that a $1 \%$ fall in the TC reduced the $\mathrm{CAD}$ risk by $2 \%{ }^{[8]}$. Helsinki heart study concluded that a mean $12 \%$ rise in HDL cholesterol and an $11 \%$ fall in LDL cholesterol were both correlated with a $34 \%$ decline in CAD. This study was undertaken with the aim to assess the lipid patterns and association of AIP with $\mathrm{HbA1c}$ in diabetes patients.

\section{Material and Methods}

This study conducted in the Department of Medicine, India from October 2018 to January 2020, after taking the approval of the protocol review committee and institutional ethics committee. After taking informed consent detailed history was taken from the patient or the relatives. The technique, risks, benefits, results and associated complications of the procedure were discussed with all patients.

\section{Methodology}

Total 160 diagnosed cases of DM type 2 in the age group of 20-70 years, attending the diabetic OPD and admitted to the medicine wards. Patients with complications like retinopathy, nephropathy, history of heart disease, hepatic disorders or any other chronic illness were excluded from the study. The patients were divided into 2 groups depending on the levels of glycated hemoglobin (HbA1c); good glycemic control was defined as $\mathrm{HbA1c}<7.0 \%(<53$ $\mathrm{mmol} / \mathrm{mol}$ ) and poor glycemic control as $\mathrm{HbA} 1 \mathrm{c}>7.0 \%$ (> $53 \mathrm{mmol} / \mathrm{mol}$ ). 9 Venous blood samples from all the subjects were collected after at least 6 hours fasting and analyzed for fasting plasma glucose (FPG), 2 hours post prandial glucose levels $(2 \mathrm{hPG})$, serum total cholesterol (TC), triglycerides (TG) using kit based method on autoanalyser XL-640 Erba. HDL-C was estimated by kit based on precipitation method10 \& Very low density lipoprotein cholesterol (VLDL-C) \& LDL-C analysis was done by applying Frieldwald's formula11 i.e. VLDL-C $=\mathrm{TG} / 5$ where $\mathrm{TG}$ is less than $400 \mathrm{mg} / \mathrm{dl}$, LDL-C was calculated as: TC - (HDLC+VLDL-C). HbA1c was calculated by Ion Exchange Resin Method. The atherogenic index of plasma (AIP) was calculated by the formula base 10 logarithm of the ratio of TG to HDL-C.12 For serum lipid reference level, National Cholesterol Education Programme (NCEP) Adult Treatment
Panel III (ATP III) guideline was referred. According to NCEP-ATPIII guidelines, hypercholesterolemia is defined as $\mathrm{TC}>200 \mathrm{mg} / \mathrm{dl}$, high LDL-C with a value $>100 \mathrm{mg} / \mathrm{dl}$, hypertriglyceridemia as TG $>150 \mathrm{mg} / \mathrm{dl}$ and low HDL-C with a value $<40 \mathrm{mg} / \mathrm{dl}$. Dyslipidemia was defined by presence of one or more than one abnormal serum lipid concentration ${ }^{[13]}$.

\section{Statistical Analysis}

Demographic and lipid parameters were presented as Mean \pm SD. Categorical variables were expressed in actual numbers and percentage. Demographic and lipid parameters were compared by performing unpaired t-test. Categorical variables were compared by performing chi-square test. $\mathrm{P}$ value $<0.05$ was considered as statistically significant. Statistical software STATA version 12.0 was used for data analysis.

\section{Results}

Out of the 160 patients studied, there were 70 males and 90 females. The maximum number of patients was in the age group of 50-60 years. Table 1 shows the comparison of studied parameters between the good and poor glycemic control group. The levels of FBG, TC, TG,VLDL-C LDL-C, $\mathrm{HbA1c}$, AIP, ratios of TC/HDL-C and LDL-C/HDL-C are increased, while the levels of HDL-C are decreased in patients with $\mathrm{HbA1c}>7 \%$ as compared to patients with $\mathrm{HbA} 1 \mathrm{c} \leq 7 \%$ and these values are highly significant. The patients with $\mathrm{HbA1c}>7 \%$ had dyslipidemia as the most prominent feature in the lipid profile. This shows that glycemic control is extremely necessary to curb the future risk of cardiovascular disease, which can be contributed to the atherogenic lipid profile.

Table 1: Demographic and Lipid parameters categorized by patients glycemic control

\begin{tabular}{|c|c|c|c|}
\hline \multirow{2}{*}{ Parameters } & \multicolumn{2}{|c|}{ Glycated haemoglobin } & \multirow{2}{*}{ p-value } \\
\cline { 2 - 3 } & $\mathbf{5 7 . 0 \%}$ & $\mathbf{7 7 . 0 \%}$ & \\
\hline Age in years & $50.37 \pm 8.47$ & $58.75 \pm 7.98$ & $<0.0001$ \\
\hline FPG & $159.87 \pm 40.34$ & $191.24 \pm 47.23$ & $<0.0001$ \\
\hline 2hPG & $213.87 \pm 62.38$ & $252.10 \pm 56.32$ & 0.0002 \\
\hline TC & $175.89 \pm 24.97$ & $225.97 \pm 43.58$ & $<0.0001$ \\
\hline TG & $151.22 \pm 33.34$ & $225.39 \pm 52.31$ & $<0.0001$ \\
\hline VLDL-C & $32.14 \pm 5.97$ & $45.12 \pm 10.22$ & $<0.0001$ \\
\hline HDL-C & $55.78 \pm 12.32$ & $49.25 \pm 7.78$ & $<0.0001$ \\
\hline LDL-C & $88.97 \pm 17.35$ & $132.34 \pm 39.98$ & $<0.0001$ \\
\hline AIP & $0.47 \pm 0.21$ & $0.68 \pm 0.19$ & $<0.0001$ \\
\hline TC/HDL-C & $3.49 \pm 0.63$ & $4.98 \pm 1.58$ & $<0.0001$ \\
\hline LDL-C/HDL- & $1.77 \pm 0.53$ & $2.89 \pm 126$ & $<0.0001$ \\
\hline C & & & \\
\hline
\end{tabular}

$\mathrm{P}<0.001$ - highly significant

Table 2 shows direct and significant correlation of $\mathrm{HbA1c}$ with FBG, 2hPG. It also shows direct and highly significant correlation of HbA1c with TC, TG, LDL-C, TC/HDL-C, LDL-C/HDL-C and AIP, and inverse correlation between $\mathrm{HbA} 1 \mathrm{c}$ and HDL.

Table 2: Correlation of HbA1c with FBG, 2hPG and lipid parameters

\begin{tabular}{|c|c|c|}
\hline Parameters & R-value & p-value \\
\hline FPG & 0.50 & $<0.0001$ \\
\hline 2hPG & 0.41 & $<0.0001$ \\
\hline TC & 0.67 & $<0.0001$ \\
\hline
\end{tabular}




\begin{tabular}{|c|c|c|}
\hline TG & 0.71 & $<0.0001$ \\
\hline VLDL-C & 0.70 & $<0.0001$ \\
\hline HDL-C & -0.38 & $<0.0001$ \\
\hline LDL-C & 0.57 & $<0.0001$ \\
\hline AIP & 0.72 & $<0.0001$ \\
\hline TC/HDL-C & 0.69 & $<0.0001$ \\
\hline LDL/HDL-C & 0.59 & $<0.0001$ \\
\hline
\end{tabular}

$\mathrm{P}<0.001-$ highly significant

Table 3: Association of AIP and $\mathrm{HbA} 1 \mathrm{c}$

\begin{tabular}{|c|c|c|c|}
\hline AIP & $\leq 7$ & $>7$ & \multirow{2}{*}{ p-value } \\
\hline$<0.11$ ( low risk) & 0 & 2 & \multirow{2}{*}{0.021} \\
\hline $0.11-0.22$ (Intermediate risk) & 4 & 0 & \multirow{2}{*}{$>0.22$ ( High risk ) } \\
\hline
\end{tabular}

$\mathrm{P}<0.05-\mathrm{S}$ (significant

Table 3 shows that patients with $\mathrm{HbA} 1 \mathrm{c}>7 \%$ were more prone to cardiovascular risk as their levels of AIP fall in the high risk group i.e AIP $>0.22$ and the association was highly significant proving that glycemic control contributes significantly to the future risk of cardiovascular problems. The lipid parameters of patients with $\mathrm{HbA} 1 \mathrm{c}<7 \%$ were in the normal range but the AIP levels still fell in the risk group.

\section{Discussion}

Insulin impacts the liver Apo lipoprotein production which regulates the enzymatic activity of lipoprotein lipase and Cholesterol ester transport protein. These could be the likely causes of dyslipidemia in Diabetes mellitus as reported by Goldberg ${ }^{[14]}$. Over and above this, insulin deficiency also reduces the activity of hepatic lipase and several other steps in the production of biologically active lipoprotein lipase may also be altered in DM ${ }^{[15]}$. A number of studies using tracer kinetics in humans have demonstrated that liver production of Apo lipoprotein B (apoB), the major protein component of VLDL and LDL, is increased in type 2 diabetes. Increased lipolysis in adipocytes due to poor insulinization results in increased fatty acid release from fat cells. The ensuing increase in fatty acid transport to the liver, which is a common abnormality seen in insulinresistant diabetes, may cause an increase in VLDL secretion ${ }^{[16]}$. A second regulatory process may be a direct effect of insulin on liver production of apoB and other proteins involved in degradation of circulating lipoproteins. In some studies insulin directly increased degradation of newly synthesized apoB ${ }^{[17]}$. Therefore, insulin deficiency or hepatic insulin resistance may increase the secretion of apoB, in turn increasing LDL-C and VLDL-C levels. Our study shows direct and significant correlation of $\mathrm{HbAlc}$ with FBG, 2 hPG, as also shown by previous studies ${ }^{[18,19]}$. Khan HA et al. also found similar correlations of $\mathrm{HbA} 1 \mathrm{c}$ with TC, TG, LDL-C and HDL-C ${ }^{[20]}$. We also found a direct and significant correlation of $\mathrm{HbAlc}$ with AIP, TC/HDL-C and LDL-C/HDL-C ratio. Thus the severity of dyslipidemia increases in patients with increased $\mathrm{HbAlc}$ value. Elevated levels of $\mathrm{HbAlc}$ and dyslipidemia are independent risk factors of cardiovascular diseases and hence, diabetic patients with elevated HbA1c and dyslipidemia are considered as high risk group for cardiovascular disease. Improving glycemic control can reduce the risk of cardiovascular events in diabetes. in our study patients with $\mathrm{HbAlc}>7 \%$ were more prone to cardiovascular risk as their levels of AIP fall in the high risk group i.e AIP > 0.22 and the association was highly significant proving that glycemic control contributes significantly to the future risk of cardiovascular problems. Dobiasova et al. ${ }^{[21]}$ was observed the patients with type 2 diabetes with poor glycemic control have the highest AIP. Dobiasova et al. also observed a higher FERHDL compared with no diabetic individuals ${ }^{[21]}$. We hypothesize that even if the lipid profile appears to be in normal range, the AIP levels when calculated can be in the risk group emphasising the calculation of this simple ratio every time a lipid profile is asked for. AIP, therefore, reflects the delicate metabolic interactions within the whole lipoprotein complex ${ }^{[21]}$.

In addition, they are more likely to have a predominance of small, dense LDL particles compared with no diabetic controls ${ }^{[22]}$. All of these factors suggest that AIP is a suitable marker for plasma atherogenicity and cardiovascular risk in patients with type 2 diabetes. Earlier studies targeted total cholesterol and HDL-C levels to reduce the risk for CVD, but the levels of triglyceride were ignored as a contributor towards this risk. Since Gaziano et al. reported that "the ratio of triglycerides to HDL was a strong predictor of myocardial infarction [23], additional findings have been made regarding relationship between HDL-C and TGs. Tan et al. ${ }^{[12]}$ compared the results of AIP analysis with those of a TG/HDL-C ratio analysis used in another study ${ }^{[24]}$ The $\mathrm{P}$ values for AIP were consistently lower than those for TG/HDLC. Although an independent, inverse relationship between HDL-C and cardiovascular risk has been demonstrated beyond any doubt, the contribution of TGs to cardiovascular risk has been underestimated. This may have been attributable to the high variability of plasma TG concentrations (which decreases the statistical significance of assessments), the lack of information on the role of TGs in biochemical mechanisms, or the incessant efforts to find an atherogenic marker independent of other lipids. In reality, any therapeutic hypolipidemic intervention leads to bigger or smaller changes in the spectrum of plasma lipids and Apo proteins, including changes in lipoprotein particle sizes and changes in cholesterol esterification and lipolytic rates ${ }^{[25]}$. Thus, TGs play the role of a regulator of lipoprotein interactions and not the role of an independent risk marker. This claim is supported by evidence that an increased plasma concentration of TGs is associated with (a) an increased incidence of coronary artery disease, (CAD) ${ }^{[26]}$ (b) an increased population of small, dense LDLs and (c) enhanced cholesteryl ester (CE) mass transfer from HDL to Apo lipoprotein B (apoB)-containing lipoproteins ${ }^{[27]}$.

\section{Conclusion}

It can be concluded from the present study that patients with poor glycemic control have a atherogenic lipid profile and Glycated haemoglobin predicts dyslipidemia and atherogenicity. Atherogenic index of plasma, is an independent parameter which correlates very significantly with the cardiovascular risk. This parameter is easy to calculate every time a lipid profile is asked for, so that the cardiovascular risk of the patient can be assessed.

\section{Reference}

1. Hameed IK, Abed BA, Rashid NF. Glycated hemoglobin as a dual marker associated between $\mathrm{HbA} 1 \mathrm{c}$ and dyslipidemia in type 2 diabetic patients, J Fac Med Baghdad. 2012;54:88-92. 
2. Mahato RV, Gyawali P, Raut PP, Regmi P, Singh KP, Pandeya DR et al. Associated between glycemic control and serum lipid Profile in type 2 diabetic patients: Glycated hemoglobin as a dual biomarker Biomedical research. 2011;22(3):375-80.

3. Sultan A, Thuan JF, Avignon. A Primary prevention of cardiovascular events and type 2 diabetes: should we prioritize our interventions? Diabetes Metab. 2006;32:559-67.

4. Amini M, Horri N, Zare M, Haghighi S, Hosseini SM, Aminorroaya A et al. People with impaired glucose tolerance and impaired fasting glucose are similarly susceptible to cardiovascular disease: a study in firstdegree relatives of type 2 diabetic patients Ann Nutr Metab. 2010;56(4):267-72.

5. American diabetes association. Implications of the United Kingdom prospective diabetes study Diabetes care. 2003;26(Suppl. 1):28-32.

6. Campbell RK. Type 2 diabetes: Where we are today: An overview of disease burden, current treatments, and treatment strategies, J Am Pharm Assoc. 2009;49(Suppl 1):S3-9.

7. Wilson PWF, D' Agostino RB, Levy D, Belanger AM, Silbershatz H, Kannel WB. Prediction of coronary heart disease using risk factor categories Circulation. 1998;97(18):1837-47.

8. The Lipid Research Clinics Coronary Primary Prevention Trial results I. Reduction in incidence of coronary heart disease JAMA. 1984;251(3):351-64

9. Position statement, Standards of Medical Care in Diabetes - 2012, Diabetes Care. 2012;35(1):S11-S63.

10. HDL-cholesterol reagent set [Kit insert]. Thane (India): Accurex Biomedical Pvt. Ltd, 2010.

11. Rifai N, Warnick GR. Lipids, lipoproteins, Apo lipoproteins, and other cardiovascular risk factors. In: Burtis CA, Ashwood ER, Bruns DE, editors. Tietz textbook of clinical chemistry and molecular diagnostics 4th ed New Delhi: Saunders Elsevier, 2006, 903-81.

12. Tan MH, Johns D, Glazer NB. Pioglitazone reduces atherogenic index of plasma in patients with type 2 diabetes Clin Chem. 2004;50:1184-88.

13. Third Report of the Expert Panel on Detection, Evaluation, and Treatment of High Blood Cholesterol in Adults (Adult Treatment Panel III.

http://www.nhlbi.nih.gov/guidelines/cholesterol/atp3ful 1.pdf

14. Goldberg IJ. Lipoprotein lipase and lipolysis: central roles in lipoprotein metabolism and atherogenesis, J Lipid Res. 1996;37:693-707.

15. Tavangar K, Murata Y, Pedersen ME, Goers JF, Hoffman AR, Kraemer FB. Regulation of lipoprotein lipase in the diabetic rat, J Clin Invest. 1992;90:1672-1678.

16. IRA J. Goldberg. Diabetic Dyslipidemia: Causes and Consequences J Clin endocrinology \& Metabolism. 2001;86:965-970.

17. Sparks JD, Sparks CE. Insulin modulation of hepatic synthesis and secretion of Apo lipoprotein B by rat hepatocytes, J Biol Chem. 1990;265:8854-8862

18. Rosediani M, Azidah A K, Mafauzy M. Correlation between fasting plasma glucose, post prandial glucose and glycated hemoglobin and fructose amine Med J. Malaysia. 2006;61:67-71.
19. Ito C, Maeda R, Ishida S, Sasaki H, Harada H. Correlation among fasting plasma glucose, two hour plasma glucose levels in OGTT and HbA1c Diabetes Res Clin Pract. 2000;50:225-30.

20. Khan H A, Sobki S H, Khan S A. Association between glycemic control and serum lipid profile in type 2 diabetic patients: $\mathrm{HbA1c}$ predicts dyslipidemia Clin Exp. Med. 2007;7:24-29.

21. Dobiasova M, Frohlich J, The plasma parameter log (TG/HDL-C) as an atherogenic index: correlation with lipoprotein particle size and esterification rate in apoBlipoprotein-depleted plasma (FER (HDL) Clin Biochem. 2001;34(7):583-588.

22. Feingold KR, Grunfeld C, Pang M, Doerrler W, Krauss RM. LDL subclass phenotypes and triglyceride metabolism in non insulin dependent diabetics Arterioscler Thromb. 1992;12:1496-502.

23. Gaziano JM, Hennekens CH, O’Donnell CJ, Breslow JL, Buring JE. Fasting triglycerides, high-density lipoprotein and risk of myocardial infarction circulation 1997;96:2520-5.

24. Lehto S, Ronnemaa T, Pyorala K, Laakso M. Cardiovascular risk factors clustering with endogenous hyperinsulinaemia predict death from coronary heart disease in patients with type II diabetes Diabetologia 2000;43:148-55.

25. Dobiasova. Implications of the AIP Clinical Chemistry. 2004;50(7):1113-1115.

26. Hokanson JE, Austin MA. Plasma triglyceride level is a risk factor to cardiovascular disease independent of high-density lipoprotein cholesterol level: a metaanalysis of population based prospective studies $\mathbf{J}$ Cardiovasc Risk. 1996;3:213-9.

27. Gue'rin M, Le Goff W, Lassel TS, Van Tol A, Steiner G, Chapman MJ. Proatherogenic role of elevated CE transfer from HDL to VLDL1 and dense LDL in type 2 diabetes Arterioscler Thromb Vasc Biol. 2001;21:2829. 